\title{
High-Pressure Tactic: Colonic Manometry in Chronic Constipation
}

\author{
Ji-Hong Chen ${ }^{1}$ - Jan D. Huizinga ${ }^{2}$
}

Published online: 7 June 2018

(c) Springer Science+Business Media, LLC, part of Springer Nature 2018

Chronic refractory constipation is a diagnostic and therapeutic challenge for adult and pediatric gastroenterologists and for affected patients. In the search for a correct diagnosis and optimal treatment options, many diagnostic tests can be performed and many therapeutic treatments explored. When minimal response to stimulant and osmotic laxatives is noted, colonic manometry appears to be a logical and recommended next step to diagnose suspected abnormal colonic motility [1]. In comparison with children, colonic manometry is rarely pursued in adults, and is available only in limited centers, and deemed of uncertain clinical relevance. Colonic manometry, as a clinical service, as opposed to a research procedure, is aimed at determining whether high-amplitude propagating contractions (HAPC) are generated under baseline conditions, in response to a meal, and in response to bisacodyl given in the proximal colon. We prefer to call the HAPC motor pattern a high-amplitude propagating pressure wave (HAPW) [2,3] since it is important to acknowledge that pressure is measured and that pressure patterns are not equivalent to muscular contraction patterns [4]. The HAPW is investigated since it is believed that this contraction pattern is essential for satisfactory defecation, although HAPWs are likely primarily essential for the transit of content in anal direction, they are also involved in the preparatory phase of defecation but may not be commonly related to the act of defecation [5]. When patients are refractory to medical treatments, including stimulant laxatives,

Jan D. Huizinga

huizinga@mcmaster.ca

Ji-Hong Chen

chen338@mcmaster.ca

1 Department of Medicine-Gastroenterology, Farncombe Family Digestive Health Research Institute, McMaster University, HSC-3V48, 1200 Main Street West, Hamilton, ON L8N 3Z5, Canada

2 Farncombe Family Digestive Health Research Institute, McMaster University, HSC-3N8E, 1200 Main Street West, Hamilton, ON L8N 3Z5, Canada the suspicion is that the colon cannot generate HAPWs and often the diagnosis of "inert colon" is proposed.

Bassotti and coworkers showed that the colon of a subgroup of adult patients deemed to have an inert colon did respond to bisacodyl with one or more HAPWs (on average 1.8 over $24 \mathrm{~h}$ ) which indicates that the colon has healthy enough musculature and innervation to produce this essential motor pattern [6]. In this issue of Digestive Diseases and Sciences [7], a group from the same institution determined whether the occurrence of HAPWs during bisacodyl testing can predict the response to further therapeutic efforts. They found that aggressive pharmacological therapy was successful in all patients that showed bisacodyl-induced HAPWs, thus avoiding surgery, whereas most patients who did not respond to bisacodyl also did not respond to the aggressive medical therapy, subsequently undergoing subtotal colectomy.

One issue with the above strategy of operating on patients that fail the bisacodyl test is that the HAPW, customarily the only colonic motor pattern considered with this test, is a rare occurrence, even in healthy subjects. Evaluating only HAPWs does not provide a complete assessment of colonic motor function, let alone give full insight into the pathophysiology of chronic constipation in adults and children. Also, in the study by Battaglia et al. [7], six patients who showed no HAPWs during the test appeared to respond well to the aggressive use of medications. Although the absence of HAPWs during the bisacodyl test seems to provide evidence of neurogenic and possibly myogenic impairment, other factors could be contributory such as bacterial dysbiosis that might prevent bisacodyl from being transformed into its active metabolite [bis-( $p$-hydroxyphenyl) pyridyl-2 methane; (BHPM)]. It is also important to allow enough time for bisacodyl to evoke activity. In our experience, luminal bisacodyl acts within $10 \mathrm{~min}$, but it may take longer than expected; in the study by Battaglia et al. [7], it took an average of $40 \mathrm{~min}$.

If bisacodyl given in the proximal colon induces HAPWs but the HAPWs are not present at baseline and are not induced by a meal, it can be interpreted that 
pharmacological stimulation of sensory nerves in the proximal colon can elicit an essential propulsive motor pattern that promotes colonic transit; it does not necessarily suggest a normal defecation reflex which usually occurs in response to a rectal stimulation. There is also no assurance that HAPWs will be evoked by physiological colonic distension or luminal metabolites. The precise mechanism of action of bisacodyl given in the proximal colon is not fully clarified; it is not certain whether the action is mediated by 5-hydroxytryptamine (5-HT) release from enterochromaffin cells and whether both intrinsic nerves and extrinsic vagal nerves are involved. Patients who respond to bisacodyl with the propagation of HAPWs measured during colonic manometry may not have a satisfactory evacuation when oral bisacodyl is used therapeutically. The latter may be due to upper GI dysmotility preventing the achievement of adequate colonic bisacodyl concentrations or due to adverse effects such as nausea and vomiting, or it may be due to dysbiosis or lack of appropriate brush border enzymes preventing bisacodyl breakdown to its active compound.

Battaglia et al. [7] convincingly show that a positive manometry test may guide the treatment of constipation; they proceed with aggressive pharmacological therapy. Nonetheless, it may be advantageous to explore a different treatment in patients that have spontaneous and mealinduced HAPWs, compared to those patients that only show HAPWs following the bisacodyl challenge. In the study by Battaglia et al. [7], seven patients showed 1-3 HAPWs in response to a meal, and only five patients showed a response to bisacodyl in the absence of any spontaneous or mealinduced HAPWs. The presence of a normal HAPW response to a meal suggests a normal musculature, a normal intrinsic nervous system that guides the HAPW throughout most of the colon, and normal responses mediated by intrinsic and extrinsic sensory and motor neurons to facilitate the gastrocolic reflex. In these cases, the original diagnosis of "inert colon" has to be completely abandoned. The presence of a normal gastrocolic reflex, that is, normal motor patterns throughout the colon in response to a meal, in the absence of outlet obstruction, may signify the ability of the colon to function normally; therefore, chronic stimulant laxative use should be avoided. In our study conducted in China [3], some patients with severe constipation who were diagnosed with inert colon but showed normal responses to a meal challenge, changed their perception of their colonic function, displaying increased enthusiasm to explore dietary and lifestyle modifications with less use of laxatives. During manometry, the patients can observe the colonic motor patterns on a screen, resulting in a greater awareness of the functioning of their colon and they can observe responses to a meal; hence, colonic manometry in this instance provides biofeedback.
Battaglia et al. [7] suggest that a limitation of their study is that with the replacement of water-perfused catheters by solid-state catheters, intraluminal perfusion of bisacodyl in the proximal colon cannot be performed. Yet, bisacodyl can be given rectally, although differences in mechanism of action between proximal and rectal bisacodyl action need to be studied, since the extrinsic sensory nerves in the proximal colon compared to the rectum have their cell bodies in different locations and are communicating with different motor neurons.

Although the bisacodyl test is very useful, as shown by Battaglia et al. [7], the test performed with low-resolution manometry does not provide a comprehensive account of colon function. The average number of HAPWs in the 24-h study period was $<2$, which emphasizes how little colonic motor activity is actually analyzed. Although the development of an HAPW that travels along the entire colon confirms that the musculature and the enteric nervous system throughout the colon are capable of producing an HAPW, it does not capture all features of normal bowel movements, which involve a host of other motor patterns, and activities of the rectum and anal sphincters. These concerns may be alleviated by high-resolution colonic manometry (HRCM), since many more sensors are placed throughout the entire colon, ideally at $1 \mathrm{~cm}$ spacing, which enables a far superior assessment of motor patterns compared to low-resolution manometry. This is much more important for the colon compared to the esophagus since the colonic motor patterns are far more varied and complex. Recent HRCM studies $[3,8]$ have shown a variety of motor patterns that are of great importance in understanding motor functions of the colon. In addition to HAPWs (Fig. 1), simultaneous pressure waves (SPWs), already reported by Rao using low-resolution manometry [9], are now better characterized by HRCM and are shown to be associated with anal sphincter relaxation and gas expulsion [3, 8]. Furthermore, motor patterns are identified that are associated with absorption and stool formation such as haustral boundary activity and erratic pressure patterns inside the haustrum, a segmentation motor pattern [3]. In the coming years, the further identification of motor patterns, their underlying mechanisms, elucidating responses to a variety of stimuli and their clinical interpretation will progress. Mechanical distension and a meal are physiological stimuli, and when a normal response to these stimuli is observed, the possibility of recovery to normal functioning can be pursued in patients with functional constipation.

Nevertheless, much is still to be learned from HRCM. Since pressure patterns are not equivalent to contraction patterns, more knowledge regarding the relationships between pressure and contraction is needed in order to correctly interpret HRCM results. We recently learned through a study in the rabbit colon that simultaneous pressure waves appear to be initiated by fast-propagating circumferential circular 


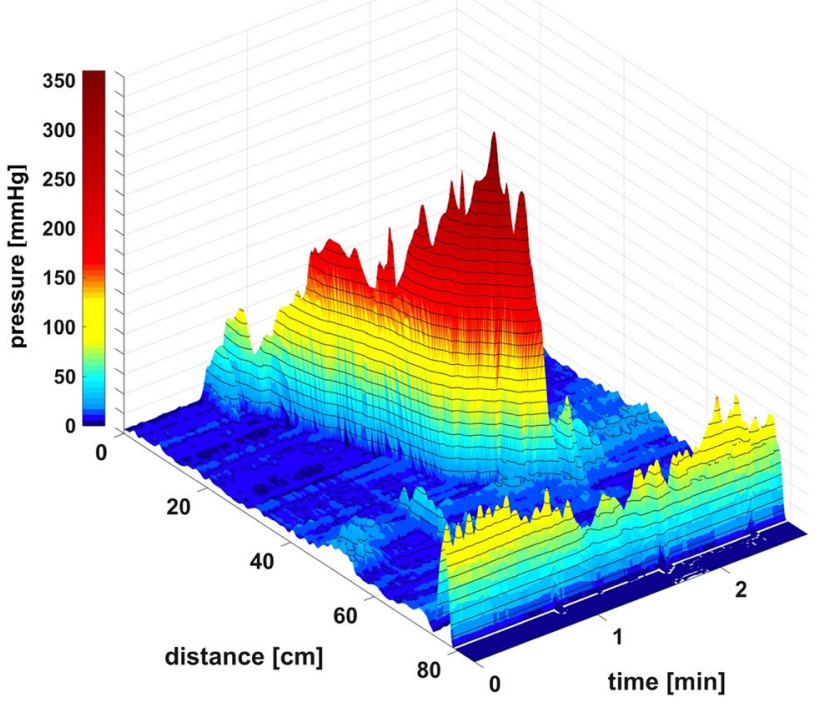

Fig. 1 A high-amplitude propagating pressure wave (HAPW) in a healthy human adult colon captured by high-resolution colonic manometry (HRCM). A water-perfused catheter with 84 sensors, $1 \mathrm{~cm}$ apart, was used [11]. The HAPW travels from the ascending colon to the descending colon. The anal sphincter remains largely closed and shows some rhythmic pressure activity; in this case, the HAPW can move content in the anal direction without evoking a full defecation reflex, which would involve anal sphincter relaxation.

muscle contractions [4]. With HRCM, retrograde cyclic motor patterns are recognized in the distal colon as part of the mechanism to maintain continence; abnormalities in this pattern may be related to the pathophysiology of constipation [10]. HRCM enables assessment of relationships between colonic motor patterns and rectal and anal activity, which is essential for full understanding of defecation disorders. HRCM will facilitate the diagnosis of refractory chronic constipation based on motor pattern assessment when the colon is challenged with pharmacological and physiological stimuli, instead of the diagnosis being based solely on symptoms and the narrative of the patient related to their bowel habits and their experience with medication.

Acknowledgments The authors gratefully acknowledge the support of the Farncombe Family Digestive Health Research Institute.

\section{Compliance with ethical standards}

Conflict of interest The authors declare that they have no conflict of interest.

\section{References}

1. Camilleri M, Bharucha AE, di Lorenzo C, et al. American Neurogastroenterology and Motility Society consensus statement on intraluminal measurement of gastrointestinal and colonic motility in clinical practice. Neurogastroenterol Motil. 2008;20:1269-1282.

2. De Schryver AM, Samsom M, Smout AJ. Effects of a meal and bisacodyl on colonic motility in healthy volunteers and patients with slow-transit constipation. Dig Dis Sci. 2003;48:1206-1212. https://doi.org/10.1023/A:1024178303076.

3. Chen J-H, Yu Y, Yang Z, et al. Intraluminal pressure patterns in the human colon assessed by high-resolution manometry. Sci Rep. 2017;7:41436. https://doi.org/10.1038/srep41436.

4. Quan X, Yang Z, Xue M, Chen J-H, Huizinga JD. Relationships between motor patterns and intraluminal pressure in the 3-taeniated proximal colon of the rabbit. Sci Rep. 2017;7:42293. https:// doi.org/10.1038/srep42293.

5. Bampton PA, Dinning PG, Kennedy ML, Lubowski DZ, deCarle D, Cook IJ. Spatial and temporal organization of pressure patterns throughout the unprepared colon during spontaneous defecation. Am J Gastroenterol. 2000;95:1027-1035.

6. Bassotti G, Chiarioni G, Germani U, Battaglia E, Vantini I, Morelli A. Endoluminal instillation of bisacodyl in patients with severe (slow transit type) constipation is useful to test residual colonic propulsive activity. Digestion. 1999;60:69-73.

7. Battaglia E, Grassini M, Pina Dore M, Bassotti G. Usefulness of biscodyl testing on therapeutic outcomes in refractory constipation. Dig Dis Sci. (Epub ahead of print). https://doi.org/10.1007/ s10620-018-4988-5.

8. Corsetti M, Pagliaro G, Demedts I, et al. Pan-colonic pressurizations associated with relaxation of the anal sphincter in health and disease: a new colonic motor pattern identified using highresolution manometry. Am J Gastroenterol. 2017;112:479-489.

9. Rao SS, Sadeghi P, Beaty J, Kavlock R, Ackerson K. Ambulatory 24-h colonic manometry in healthy humans. Am J Physiol Gastrointest Liver Physiol. 2001;280:G629-G639.

10. Dinning PG, Wiklendt L, Maslen L, et al. Colonic motor abnormalities in slow transit constipation defined by high resolution, fibre-optic manometry. Neurogastroenterol Motil. 2015;27:379-388.

11. Chen J-H, Ratcliffe E, Yuan Y, et al. Characterizing simultaneous pressure waves in the human colon by high resolution manometry. J Can Assoc Gastroenterol. 2018;1:409-410. 